\title{
openheart Review of Irish patients meeting ST elevation criteria during the COVID-19 pandemic
}

Luke Byrne (D) , Roisin Gardiner, Patrick Devitt, Caleb Powell, Richard Armstrong, Sinead Teehan, Stephen O'Connor

To cite: Byrne L, Gardiner R, Devitt $\mathrm{P}$, et al. Review of Irish patients meeting ST elevation criteria during the COVID-19 pandemic. Open Heart 2021;8:e001716. doi:10.1136/ openhrt-2021-001716

Received 9 May 2021 Accepted 13 July 2021
Check for updates

\section{(C) Author(s) (or their} employer(s)) 2021. Re-use permitted under CC BY-NC. No commercial re-use. See rights and permissions. Published by BMJ.

Department of Cardiology, Saint James's Hospital, Dublin, Ireland

Correspondence to

Dr Luke Byrne; lubyrne@tcd.ie

\section{ABSTRACT}

Introduction The COVID-19 pandemic has seen the introduction of important public health measures to minimise the spread of the virus. We aim to identify the impact government restrictions and hospital-based infection control procedures on ST elevation myocardial infarction (STEMI) care during the COVID-19 pandemic. Methods Patients meeting ST elevation criteria and undergoing primary percutaneous coronary intervention from 27 March 2020, the day initial national lockdown measures were announced in Ireland, were included in the study. Patients presenting after the lockdown period, from 18 May to 31 June 2020, were also examined. Time from symptom onset to first medical contact (FMC), transfer time and time of wire cross was noted. Additionally, patient characteristics, left ventricular ejection fraction, mortality and biochemical parameters were documented. Outcomes and characteristics were compared against a control group of patients meeting ST elevation criteria during the month of January.

Results A total of 42 patients presented with STEMI during the lockdown period. A significant increase in total ischaemic time (TIT) was noted versus controls ( 8.81 hours $( \pm 16.4)$ vs 2.99 hours $( \pm 1.39), p=0.03)$, with increases driven largely by delays in seeking FMC (7.13 hours $( \pm 16.4)$ vs 1.98 hours $( \pm 1.46), p=0.049)$. TIT remained significantly elevated during the postlockdown period (6.1 hours $( \pm 5.3), p=0.05)$, however, an improvement in patient delays was seen versus the control group (3.99hours $( \pm 4.5), p=0.06)$. There was no difference seen in transfer times and door to wire cross time during lockdown, however, a significant increase in transfer times was seen postlockdown versus controls ( 1.81 hours $( \pm 1.0)$ vs 1.1 hours $( \pm 0.87), p=0.004)$.

Conclusion A significant increase in TIT was seen during the lockdown period driven mainly by patient factors highlighting the significance of public health messages on public perception. Additionally, a significant delay in transfer times to our centre was seen postlockdown.

\section{INTRODUCTION}

The SARS-CoV-2 pandemic has caused significant public health issues, first from direct implications of viral infection, such as pneumonitis, thrombophilia and myocarditis, and second, from staffing shortages and public

\section{Key questions}

What is already known about this subject?

- Previous studies have highlighted a worrying delay in ST elevation myocardial infarction (STEMI) treatment during the COVID-19 pandemic, influenced primarily by delays in the public seeking medical attention. Most papers on this topic report on a short period of time during the initial lockdown period. Little is known on the impact of the COVID-19 pandemic on STEMI treatment during the latter phases of lockdown and into the postlockdown period.

What does this study add?

- This study highlights an important temporal relationship between the imposing, and easing of government restrictions and members of the public seeking urgent medical attention.

- Deficiencies in patient transfer to our centre were identified which resulted in further delays in STEMI management, and persisted after easing of government restrictions.

- Cardiac catheterisation laboratory infection control measures applied during the pandemic did not result in delays in STEMI treatment at any stage.

How might this impact on clinical practice?

- Further emphasis should be placed on educating the public to seek medical attention for emergency presentations during times in which government restrictions are applied.

fears with regard to hospital attendance. ${ }^{1}$ The respiratory manifestations of SARS-CoV-2 infection has been well documented across the literature, however, the implications of the pandemic on the cardiovascular health of a population are less well known. ${ }^{2}$

A report from Hong Kong noted a significant mean time delay in hospital presentation in patients with ST elevation myocardial infarction (STEMI) from $82.5 \mathrm{~min}$ at baseline to 318 min during the pandemic in a small cohort of patients. Although not reported in the paper, given the time critical nature of STEMI management, it is assumed a significant increase in patient morbidity and 
mortality may be incurred as a result. ${ }^{3}$ A recent paper published data from another primary percutaneous coronary intervention (PPCI) centre in the Ireland reported a significant increase in total ischaemic time (TIT), driven by patient delays in seeking medical attention, with further studies published from other centres in Europe reporting similar results. ${ }^{45}$ Furthermore, a British study reported startling delays in time of symptoms onset to first medical contact (FMC), with an increase in the 75 percentile from 3.4 to 13.2 hours. ${ }^{6}$ Additionally, a recent survey carried out by the European Society of Cardiology (ESC) suggested a perceived 50\% reduction in STEMI presentations globally with respondents reporting $48 \%$ of patients presented later than usual during the pandemic. ${ }^{7}$ Reports from other fields of medicine, such as stroke, where time dependent treatments are also crucial mirror these unsettling findings. ${ }^{8}$

Finally, the impact of the pandemic has had significant effects on the management of chronic coronary syndrome (CCS). One multicentre study reported a $74 \%$ reduction in number of elective invasive coronary procedures performed for patients with CCS. Additionally, there was, however, a significant increase in the proportion of acute coronary syndrome (ACS) presentations versus the previous year. Significant delays from FMC to wire cross were seen in patients with COVID-19, which was attributed to catheterisation lab preparation times. ${ }^{9}$

This study was carried out in St James' Hospital which is the largest primary PCI centre in the Ireland performing $25.8 \%$ of national cases. ${ }^{10}$ In keeping with ESC guidance, a target time from FMC to wire cross is $<90 \mathrm{~min}$, with increasing delays in revascularisation associated with poorer outcomes. $^{1112}$

COVID-19 cardiac catheterisation infection control procedures such as the use of personal protective equipment (PPE), using a dedicated COVID-19 lab and routine COVID-19 screening were implemented in our centre in line with international guidance. ${ }^{13}$ Given the concerning body of evidence being published internationally suggesting significant delays in STEMI management, we aimed to report on our experience in terms of STEMI management during, and after, the COVID-19 lockdown to identify important patient and system factors influencing care.

\section{METHODS}

\section{Patient selection}

All patients referred to our hospital undergoing coronary angiography meeting STEMI criteria eligible for inclusion in this single centre observational study. STEMI was defined as per the European Society of Cardiology guidelines for the management of acute myocardial infarction in patients presenting with ST-segment elevation. ${ }^{11}$ Those meeting ST elevation criteria for any reason other than ACS, or those in whom revascularisation was not performed were excluded from analysis. Baseline characteristics, initial and peak troponin levels, renal profile, full blood count, C reactive peptide, time of symptom onset, time of FMC, time of arrival at our centre and time of wire cross was noted. Mortality data were also collected for all patients. Patient groups were split into those presenting during the lockdown period, from 27 March 2020 to 18 May 2020, the official date restrictions were eased, and those presenting postlockdown from 18 May 2020 to 31 June $2020 .^{14}$

A control group of all revascularised STEMI patients presenting to our hospital from 1 January 2020 to 31 January 2020 were examined to determine significance. Patients presenting in February were excluded as a control group due to a subjective increase in public concern about COVID-19.

FMC was taken as the time the first positive STEMI ECG was recorded. Transfer time was taken as the delay from FMC to arrival at our PPCI centre. Patients first diagnosed in the emergency department of our centre were not included in this analysis. Door to wire cross was taken as the time from arrival at PPCI centre to wire cross.

\section{Biochemical data}

Initial and peak troponin levels were taken as the first and highest troponin measured during inpatient stay in our centre. All other biochemical data including full blood count and renal profile were taken as the first level measured.

\section{Statistical analysis}

Categorical variables are presented as percentages and examined using the $\chi^{2}$ test and Fisher's Exact test where appropriate. Continuous variables are presented as a mean $( \pm \mathrm{SD})$ or median $(\mathrm{IQR})$ and where assessed for significance using the Student's t-test. $\mathrm{P}$ values were two tailed and a $p<0.05$ was deemed significant. Data analysis was carried out on using Microsoft excel.

\section{RESULTS}

\section{Baseline demographics}

A total of 42 patients presented during the lockdown period and 39 presented postlockdown. The mean ages were similar between the three groups and, apart from an increased proportion of hypertensive patients presenting postlockdown, there was no significant difference in terms of risk factors for cardiovascular disease. An outline of baseline characteristics can be seen in table 1 .

\section{Time delays in management}

To identify any delays in STEMI management during the lockdown, those presenting during the lockdown period were compared with a control group of patients presenting during January 2020. TIT was defined as the time from symptom onset to wire cross. Mean (mean $( \pm$ SD) ) TIT during lockdown was $8.81( \pm 16.4)$ hours vs $2.99( \pm 1.39)$ hours in the control group $(\mathrm{p}=0.03)$.

This was further broken down into time from symptom onset to FMC, time from FMC to arrival at PPCI centre (transfer time) and door to wire cross. 


\begin{tabular}{lllll}
\hline Table 1 & Patient demographics & & & \\
\hline Parameter & Control $(\mathbf{n}=\mathbf{2 4})$ & Lockdown $(\mathbf{n}=\mathbf{4 2})$ & Postlockdown $(\mathbf{n}=\mathbf{3 9})$ & P value \\
\hline Mean age $( \pm$ SD) & $64.1(14.3)$ & $56.7( \pm 10.6)$ & $60.6( \pm 13.3)$ & $0.02,0.3^{*}$ \\
\hline Male (\%) & $19(79.2)$ & $27(64.3)$ & $33(80.5)$ & 0.4 \\
\hline Previous IHD (\%) & $1(4.2)$ & $2(4.8)$ & $5(12.2)$ & 0.42 \\
\hline Smoker (\%) & $17(58.3)$ & $19(45.2)$ & $24(53.3)$ & 0.13 \\
\hline Diabetes (\%) & $2(8.3)$ & $1(2.4)$ & $5(11.1)$ & 0.28 \\
\hline Hypertension (\%) & $6(25)$ & $8(19)$ & $21(46.7)$ & 0.01 \\
\hline Dyslipidaemia (\%) & $8(33.3)$ & $5(9.5)$ & $13(28.9)$ & 0.08 \\
\hline Chronic kidney disease (\%) & 0 & 0 & 0 & - \\
\hline Previous CVA/TIA (\%) & 0 & $1(2.4)$ & 0 & - \\
\hline
\end{tabular}

Data are presented as mean $( \pm \mathrm{SD})$, absolute values and percentages, as appropriate. Categorical variables were assessed for significance using $\chi^{2}$ test and Fisher's exact test where appropriate.

${ }^{*} \mathrm{P}$ value is presented as lockdown versus control and postlockdown versus control, respectively.

CVA, cerebrovascular accident; IHD, ischaemic heart disease; TIA, transient Ischaemic attack.

Mean time from symptom onset to FMC was 7.13 hours during lockdown $( \pm 16.4)$ vs 1.98 hours $( \pm 1.46)$ in the control group $(\mathrm{p}=0.049)$.

Mean time from FMC to arrival at PPCI centre, or transfer time, was noted to be 1.29 hours $( \pm 0.76)$ during lockdown vs 1.1 hours $( \pm 0.87)$ in the control group $(\mathrm{p}=0.92)$.

Finally, door to wire cross time during lockdown was 0.49 hours $( \pm 0.27)$ vs 0.41 hours $( \pm 0.14)$ in controls $(\mathrm{p}=0.18)$.

An analysis of patients presenting after the easing of government restrictions was carried out to establish a temporal relationship between national lockdown measures and STEMI presentations. TIT in the postlockdown group improved marginally versus the lockdown group, however, significant delays were still versus the control group (6.1 hours $( \pm 5.3)$ vs 2.99 hours $( \pm 1.39), \mathrm{p}=0.05)$. Mean time from symptom onset to FMC improved postlockdown to 3.9 hours $( \pm 4.5)$ $(p=0.06)$, however, transfer times were significantly elevated during this period versus the control group ( 1.81 hours $( \pm 1.0), p=0.004)$. Door to wire cross time remained stable at 0.44 hours $( \pm 0.15) \quad(p=0.6)$. An outline of important time points in STEMI management is outlined in table 2 below.
An analysis of mean TIT on a weekly basis showed the longest delays were seen in the first 3 weeks of the lockdown period (mean of 17.7 hours $( \pm 26.5)$, $\mathrm{p}=0.09$ ) . Mean TIT subsequently improved in the latter stage of lockdown and in the postlockdown period, however, remained prolonged compared with the control group. A breakdown of weekly TIT is outlined in figure 1 .

There was a numerical increase in peak troponin levels observed in the lockdown group, however, this did not reach statistical significance $(5621 \mathrm{ng} / \mathrm{L}( \pm 3468)$ vs $3803 \mathrm{ng} / \mathrm{L}( \pm 3225), \mathrm{p}=0.08)$. Additionally, initial troponin levels were similar between the two groups $(1421( \pm 1995)$ vs $1406( \pm 1523), p=0.98)$. As mean TIT was greatest during the first 3 weeks of lockdown analysis of troponin levels during this time period was carried out. Mean peak troponin during the first 3 weeks of lockdown was $7864 \mathrm{ng} / \mathrm{L}( \pm 3089)$ vs $5621 \mathrm{ng} / \mathrm{L}( \pm 3686)$ in controls $(\mathrm{p}=0.005)$. An outline of mean peak troponin levels by week can be seen in figure 2 .

There was no significant difference noted in terms of renal function, white cell count (WCC) and $\mathrm{C}$ reactive peptide at any stage. Furthermore, no difference in left ventricular ejection fraction (LVEF) was noted between the groups $(43 \%( \pm 10.7)$ vs $43.9 \%( \pm 11), p=0.9)$. Finally, there was no difference in inpatient or 30-day mortality

\begin{tabular}{|c|c|c|c|c|c|}
\hline Parameter (in hours) & Control $(n=24)$ & Lockdown $(n=42)$ & $P$ value & Postlockdown (n=39) & $P$ value \\
\hline Mean total ischaemic time $( \pm S D)$ & $2.99( \pm 1.39)$ & $8.81( \pm 16.4)$ & 0.03 & $6.1( \pm 5.3)$ & 0.05 \\
\hline Mean time from symptom onset to $\mathrm{FMC}( \pm \mathrm{SD})$ & $1.98( \pm 1.46)$ & $7.13( \pm 16.4)$ & 0.049 & $3.9( \pm 4.5)$ & 0.06 \\
\hline Mean transfer time $( \pm \mathrm{SD})$ & $1.1( \pm 0.87)$ & $1.29( \pm 0.76)$ & 0.92 & $1.81( \pm 1.0)$ & 0.004 \\
\hline Mean door to wire cross $( \pm S D)$ & $0.41( \pm 0.14)$ & $0.49( \pm 0.27)$ & 0.18 & $0.44( \pm 0.15)$ & 0.6 \\
\hline
\end{tabular}

The lockdown and postlockdown groups are compared against the control group to determine significance. Data are presented as mean $( \pm \mathrm{SD})$, absolute values and percentages, as appropriate. Categorical variables were assessed for significance using $\chi^{2}$ test and Fisher's exact test where appropriate.

FMC, first medical contact. 
Mean Total Ischaemic Time in Hours by Week

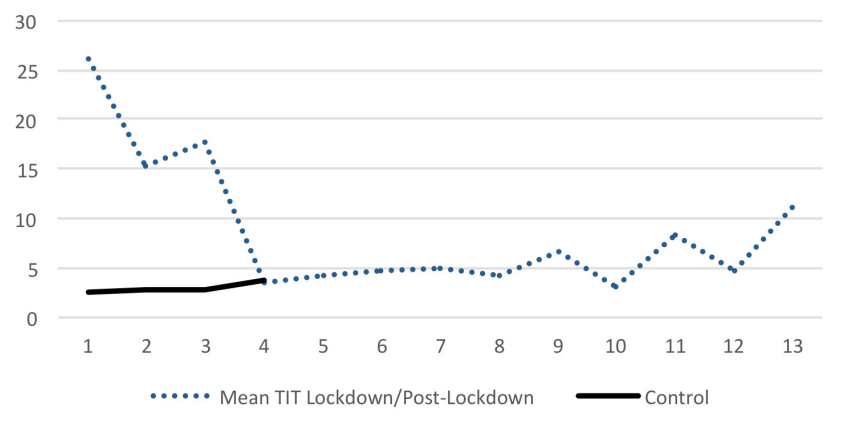

Figure 1 Outlines mean total ischaemic time (TIT) in hours on a week-by-week basis. Week 1 is taken as the week commencing on the 1 January 2020 in the control group and from 28 March 2020 in the lockdown/postlockdown group. Each week is taken as a 7 -day period from the start date in each respective group.

noted between the two groups. An outline of this data can be seen in table 3 .

\section{DISCUSSION}

During the nationwide COVID-19 lockdown, there was a significant increase in the TIT vs the control group (8.81 $( \pm 16.4)$ hours vs $2.99( \pm 1.39), \mathrm{p}=0.03)$. This was largely driven by an increase in delays from symptom onset to FMC (7.13 hours $( \pm 16.4)$ vs 1.98 hours $( \pm 1.46), p=0.049)$. These findings, in line with similar reports, highlight an important and modifiable factor impeding STEMI management during the pandemic. Increase delays in seeking medical attention or attending an emergency department is presumed to be due to increase public concern about contracting COVID-19 in the hospital setting. Although hospital attendance was advised against where possible early in the lockdown phase, certain medical emergencies such as STEMI require time critical intervention to

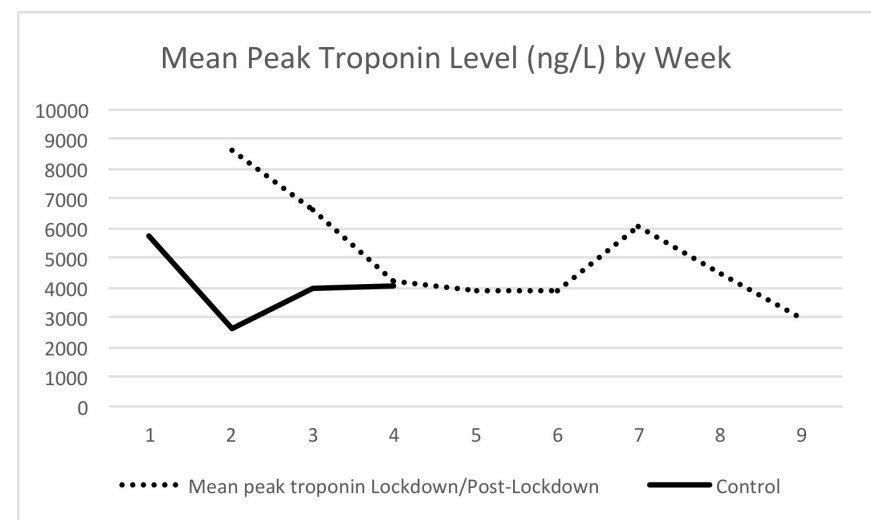

Figure 2 Outlines mean peak troponin levels in $\mathrm{ng} / \mathrm{L}$ on a week-by-week basis. Week 1 is taken as the week commencing on the 1 January 2020 in the control group and from 28 March 2020 in the lockdown/postlockdown group. Each week is taken as a 7-day period from the start date in each respective group.
Table 3 Biochemical parameters, LVEF and mortality between the two groups

\begin{tabular}{llll}
\hline Parameter $(\mathbf{E S D})$ & Control $(\mathbf{n}=\mathbf{2 4})$ & Lockdown $(\mathbf{n}=\mathbf{4 2})$ & $\mathbf{P}$ value \\
\hline $\mathrm{Hb}$ & $13.2( \pm 1.8)$ & $14.1( \pm 1.4)$ & 0.05 \\
WCC & $11.8( \pm 4.6)$ & $12.9( \pm 4.5)$ & 0.41 \\
Lymphocytes & $1.7( \pm 1.4)$ & $1.5( \pm 0.6)$ & 0.51 \\
Platelets & $242( \pm 60.5)$ & $237( \pm 58.5)$ & 0.76 \\
Creatinine & $81.7( \pm 25.1)$ & $81.9( \pm 19.6)$ & 0.97 \\
Urea & $6.3( \pm 2.9)$ & $6.6( \pm 2.8)$ & 0.72 \\
eGFR & $77.9( \pm 15.9)$ & $76.8( \pm 14.2)$ & 0.81 \\
Initial troponin & $1421( \pm 1995)$ & $1406( \pm 1523)$ & 0.98 \\
Peak troponin & $3803( \pm 3225)$ & $5621( \pm 3468)$ & 0.08 \\
CRP & $3.51( \pm 2)$ & $6.9( \pm 7.2)$ & 0.35 \\
LVEF & $43.9( \pm 11)$ & $43.3( \pm 10.7)$ & 0.9 \\
Mortality at 30 days(\%) & $2(8.3)$ & $1(2.4)$ & 0.6 \\
\hline
\end{tabular}

Data are presented as mean $( \pm S D)$, absolute values and percentages, as appropriate. Categorical variables were assessed for significance using $\chi^{2}$ test and Fisher's exact test where appropriate. CRP, $C$ reactive peptide; eGFR, estimated glomerular filtration rate; $\mathrm{Hb}$, haemoglobin; LVEF, left ventricular ejection fraction; WCC, white cell count.

prevent significant morbidity and mortality. Our public health message should be expanded to ensure timely medical attention is sought for certain medical emergencies. Patients presenting postlockdown had shorter delays in seeking medical attention even though TIT remained elevated (3.9 hours $( \pm 4.5), \mathrm{p}=0.06)$.

Despite a significant increase in TIT during lockdown, no difference in mortality was noted. A trend towards higher peak troponin levels and WCC count was noted during the lockdown period, however, this did not reach statistical significance. There was a significant increase in peak troponin noted in the first 3 weeks of lockdown, correlating with increased TIT documented during this period. There was no difference in LVEF noted between the groups.

Although a total reduction in COVID-19 cases was evident in the population at the end of the lockdown period, infection control procedures remained in place to minimise the spread of the virus. In line with other national centres, all patients presenting with STEMI were managed in full COVID-19 precautions including the use of PPE for all staff. ${ }^{13}$ There was no difference in terms of door to wire cross times seen at any stage during the study period indicating donning and doffing of PPE, and catheterisation lab cleaning between cases, did lead to delays in treatment.

Although transfer times remained stable during the lockdown period, a significant increase in transport time to our centre was noted during the postlockdown period. This finding highlights a worrying systemic factor in treatment delays of STEMI patients. The cause for this delay is not clear and outside the scope of this paper, however, increased burden on the national ambulance service and staffing shortages in the hospital setting may be causative. 
These findings, in line with other papers published on the topic, highlight the significant impact our public health message has on national STEMI care. Although other centres have reported on similar delays encountered in their own patient cohort, this paper is the first to demonstrate a clear temporal relationship between government restrictions and delays in seeking medical attention. The public should be advised to urgently seek medical help should they experience chest pain and greater emphasis should be placed on such a message during subsequent lockdown periods. Cardiac catheterisation lab infection control protocols did not result in delays in management, however, further work if needed to maximise the efficiency of patient transfers to our centre.

Limitations of this study includes a short follow-up time and a relatively small sample size. As such this study is not powered to identify a significant difference in mortality for STEMI patients presenting during, and after, the COVID-19 lockdown. Larger scale trials or meta analyses are required to quantify the mortality and morbidity impact of the pandemic on STEMI care. Furthermore, temporal changes in the number of STEMI presentations on a monthly basis should be considered when interpreting the results of this study. Additionally, data were retrospectively collected using the national ACS database rather than prospectively obtained. As such complete study datapoints could not be collected for all patients included for analysis. Finally, these data represent a single centre's experience in a localised geographical region; patient and systemic factors in different centres may produce conflicting results. However, this paper in line with others published on this topic supports a growing body of concern in delivering timely STEMI care during the COVID-19 pandemic.

Acknowledgements The authors would like to acknowledge the hard work of our institution's cardiac nurses and physiologists.

Contributors LB designed study protocol, collected study data, performed data analysis and drafted the study manuscript. RG, PD, CP and ST collected study data. RA assisted in study protocol design. S0 assisted in study protocol design and drafting the study manuscript.

Funding The authors have not declared a specific grant for this research from any funding agency in the public, commercial or not-for-profit sectors.

Competing interests None declared.

Patient consent for publication Not required.

Ethics approval This study received full ethical approval on the 4 June 2020 under the application number 20-NREC-COV-043. Ethical approval for this study was provided by the National Research Ethics Committee for COVID-19-related
Health Research (NREC COVID-19), Dublin, Ireland. The study was designed and performed in compliance with the Declaration of Helsinki.

Provenance and peer review Not commissioned; externally peer reviewed.

Data availability statement All data for the purpose of this study as stored in fully anonymised document available from the corresponding author on reasonable request.

Open access This is an open access article distributed in accordance with the Creative Commons Attribution Non Commercial (CC BY-NC 4.0) license, which permits others to distribute, remix, adapt, build upon this work non-commercially, and license their derivative works on different terms, provided the original work is properly cited, appropriate credit is given, any changes made indicated, and the use is non-commercial. See: http://creativecommons.org/licenses/by-nc/4.0/.

ORCID iD

Luke Byrne http://orcid.org/0000-0002-7389-4979

\section{REFERENCES}

1 Vlachakis PK, Tentolouris A, Kanakakis I. Concerns for management of STEMI patients in the COVID-19 era: a paradox phenomenon. $J$ Thromb Thrombolysis 2020;50:809-13.

2 Chen N, Zhou M, Dong X, et al. Epidemiological and clinical characteristics of 99 cases of 2019 novel coronavirus pneumonia in Wuhan, China: a descriptive study. Lancet 2020;395:507-13.

3 Tam C-CF, Cheung K-S, Lam S, et al. Impact of coronavirus disease 2019 (COVID-19) outbreak on ST-segment-elevation myocardial infarction care in Hong Kong, China. Circ Cardiovasc Qual Outcomes 2020;13:e006631.

4 Coughlan JJ, Chongprasertpon N, Arockiam S, et al. COVID-19 and STEMI: a snapshot analysis of presentation patterns during a pandemic. Int J Cardiol Heart Vasc 2020;30:100546.

5 Reinstadler SJ, Reindl M, Lechner I, et al. Effect of the COVID-19 pandemic on treatment delays in patients with ST-segment elevation myocardial infarction. J Clin Med 2020;9:2183.

6 Abdelaziz HK, Abdelrahman A, Nabi A, et al. Impact of COVID-19 pandemic on patients with ST-segment elevation myocardial infarction: insights from a British cardiac center. Am Heart $J$ 2020;226:45-8.

7 Pessoa-Amorim G, Camm CF, Gajendragadkar P, et al. Admission of patients with STEMI since the outbreak of the COVID-19 pandemic: a survey by the European Society of Cardiology. Eur Heart J Qual Care Clin Outcomes 2020;6:210-6.

8 Nagamine M, Chow DS, Chang PD, et al. Impact of COVID-19 on acute stroke presentation at a comprehensive stroke center. Front Neurol 2020;11:850.

9 Siudak Z, Grygier M, Wojakowski W, et al. Clinical and procedural characteristics of COVID-19 patients treated with percutaneous coronary interventions. Catheter Cardiovasc Interv 2020;96:E568-75.

10 Daly K, Jennings S. Heart attack care Ireland 2016 report of the National clinical programme for acute coronary syndrome (ACS) on standardising treatment of patients with STEMI in 20162015.

11 Van de Werf F, Staff E. ESC guidelines on the management of acute myocardial infarction in patients presenting with STEMI. Eur Heart $J$ 2008;29:2909-45.

12 De Luca G, Suryapranata H, Ottervanger JP, et al. Time delay to treatment and mortality in primary angioplasty for acute myocardial infarction: every minute of delay counts. Circulation 2004;109:1223-5.

13 Szerlip M, Anwaruddin S, Aronow HD. Considerations for cardiac catheterization laboratory procedures during the COVID-19 pandemic. Catheter Cardiovasc Interv 2020;10.

14 Ireland Go. Government of Ireland. Daily briefing on the government's response to COVID-19 - Friday 27 March 2020; 2020 [Accessed 28 Sep 2020]. 